\title{
A Study on the Class Construction and Contents by Prospective Korean Language Teachers
}

\author{
예비 한국어 교사의 수업 구성과 내용에 대한 연구 \\ Sangsoo Kim ${ }^{1}$ \\ 김상수 ${ }^{1}$ \\ ${ }^{1}$ Assistant Professor, School of Interdisciplinary, Major in Korean Language Education,
}

Tongmyong University, Korea, kospora@naver.com

\begin{abstract}
The purpose of this study was to identify the problems and failures in writing the Korean language lesson plan. For this, 50 feedback reports on the Korean language lesson plan were collected and analyzed using the Nvivo12 program. The total number of coding was 228, and coding items were reclassified according to 5 class stages. The number of coding in the Warm-up stage was 42 , there are 86 in the presentation and explanation stage, 48 in the practice stage, the use stage was 46 , and the follow up stage was 6 . The number of codings for the presentation and explanation stage was the highest with 86. The results of the study confirmed that the pre-service teachers had the most difficulty in composing the contents of the presentation and explanation stage in the process of preparing the lesson plan. In the stage of presentation and explanation, the teacher plays the biggest role in the course of the lesson, and depending on the teacher's presentation and explanation of the target item, it can have a significant impact on the progress of the next stage of the lesson. Therefore, if teacher training institutes and instructors are aware of these problems, it will be able to help prospective teachers to solve the difficulties encountered in class preparation and planning process.
\end{abstract}

Keywords: Korean Language Education, Lesson Plan, Prospective Teacher, Teaching Practice, Class Construction

요약: 이 연구는 한국어 수업 교안 작성의 문제점과 실태를 파악하는 데 목적을 두었다. 이를 위해 한국어 수업지도안에 대한 피드백 보고서 50편을 수집하였으며, NVIV012 프로그램을 사용하여 분석하였다. 분석 결과, 전체 코딩 수는 228 개로 나타났으며 코딩 항목은 5 개의 수업 단계에 따라 재분류하였다. 도입 단계의 코딩 수는 42 개, 제시와 설명 단계는 86 개, 연습 단계는 48 개, 활용 단계는 46 , 마무리 단계는 6 개로 확인되었다. 제시와 설명 단계에 대한 코딩 수가 86개로 가장 많이 나타났다. 이를 통하여 예비 교사들은 수업지도안을 작성하는 과정에서 제시와 설명 단계의 내용을 구성하는 데 가장 많은 어려움을 겪고 있는 것을 확인할 수 있었다. 제시와 설명의 단계는 수업을 진행하는 과정에서 교사의 역할이 가장 크며, 교사의 목표 항목 제시와 설명에 따라 다음 단계의 수업 진행에 상당한 영향을 미칠 수 있다. 따라서 교사 양성 기관과 교수자가 이러한 문제점을 인식한다면 예비 교사들이 수업 준비와 계획 과정에서 겪는 어려움을 해결하는 데 도움을 줄 수 있을 것이다.

Received: June 24, 2021; $1^{\text {st }}$ Review Result: August 12, 2021; $2^{\text {nd }}$ Review Result: September 27, 2021 Accepted: October 31, 2021 
핵심어: 한국어교육, 수업지도안, 예비 교사, 교육실습, 수업 구성

\section{1. 서론}

이 연구는 예비 한국어 교사의 한국어 수업 구성과 수업 운영 능력 향상에 관심을 가지고, 예비 한국어 교사가 작성한 수업지도안을 분석하여 수업 단계별 구성과 내용을 탐색하는 데 목적이 있다. 그리고 이를 통하여 한국어 교사 양성 과정의 문제점을 도출하여 한국어 교사의 전문성 향상을 위한 논의 과제를 제시하고자 한다.

한국어 교사가 되기 위해서는 전공 교육과정을 이수하는 과정을 거치게 된다. 그리고 교육과정 이수의 마지막 단계에서 '한국어교육실습' 교과목을 수강함으로써 한국어 교육 현장에 필요한 한국어 교사로서의 면모를 갖추게 된다. 즉, 예비 한국어 교사는 한국어교육실습을 통하여 교육과정 이수 과정에서 학습한 다양한 한국어 교수 학습과 관련된 정보를 교육 현장에 적용하는 연습을 하게 된다[1][2]. 이 과정에서 한국어 수업 구성과 교수에 필요한 수업지도안 작성 연습이 이루어진다[3].

예비 교사가 이수한 모든 교과목의 전문적인 내용은 한국어 교육 현장에서 실제 수업을 하는 능력으로 수렴될 수 있어야 한다. 그리고 그 능력은 현장에서 수업을 실행하기 이전에 수업지도안으로 표현할 수 있어야 한다[4]. 따라서 수업지도안은 예비 교사의 수업 실행 능력을 어느 정도 가늠할 수 있는 기준이 될 수 있다. 하지만 이러한 중요성에도 불구하고 많은 예비 한국어 교사들이 수업지도안 작성에 대한 어려움을 호소하는 경우가 많다[5][6].

수업지도안 작성의 문제점은 한국어 교육 전공 교육과정에서 찾을 수 있을 것이다. 전공 교육과정의 최종 단계에서 이수하게 되는 '한국어 교육실습' 의 경우 예비 교사들이 현장으로 나가기 이전에 이수하는 마지막 단계의 교과목이다. 해당 교과목은 실제 수업 실행과 운영에 초점을 두고 운영이 되어야 한다는 것이다. 하지만 정작 예비 교사들은 '한국어 교육실습' 교과목을 이수하는 과정에서 수업 실행을 위해 준비되어 있어야 하는 수업지도안의 구성 방법과 내용 등에 대해서 지도를 받게 된다. 즉, 전공 교육과정의 최종 단계에 이르기까지 수업지도안 작성을 비롯한 한국어 수업 구성의 방법과 내용에 대해 지도를 받지 못하는 경우가 많다는 것이다.

이에 본 연구에서는 예비 한국어 교사들이 작성한 수업지도안을 확인하고 분석하여 이들이 한국어 수업을 준비하는 과정에서 어떠한 어려움을 격고 있으며, 이러한 어려움이 향후 수업 실행 과정에서 어떠한 문제점으로 나타날 수 있는지를 살피고자 한다. 특히, 단계별 수업 구성과 내용을 탐색함으로써, 이들이 전공 교육과정을 통하여 학습한 전공 교과목에 대한 지식이 실제 현장에서 어떻게 실행될 수 있는지를 파악하고자 한다. 그리고 그 과정에서 발생할 수 있는 문제점이 무엇인지 확인하고자 한다.

\section{2. 한국어 수업 단계}

일반적으로 교실 수업은 교사와 학생 간의 상호작용을 바탕으로 이루어지는 체계적인 교수-학습의 활동 과정이다. 그리고 이 과정은 교사의 수업지도안 작성을 통하여 계획되며 구체화 된다. 숙련된 교사는 해당 교과목에서 요구하는 구체적이고 현실적인 
학습 단계와 수업 활동을 계획하는 데 능숙하여 수업 진행 과정 전체를 큰 어려움 없이 준비할 수 있다. 이러한 능숙함은 교사가 계획하는 학습 목표를 달성할 수 있고 수업이 엉뚱한 방향으로 흘러가는 것을 방지할 수 있는데, 이는 수업지도안 작성 과정을 통하여 수업의 전체적인 구성과 내용을 적절하게 계획하기 때문이다[7].

한국어 수업지도안 작성 과정에서 수업 단계의 구분은 '도입-제시/설명-연습 활용(사용)-마무리(정리)’ 의 5단계로 나누는 것이 일반적이다. 우선, ‘도입(Warmup)' 단계에서는 선수 학습한 내용을 학습자들에게 상기시키고 해당 차시의 학습 목표를 안내하고 해당 수업 주제에 대한 학습자의 흥미를 유발할 수 있도록 구성한다.

'제시(Presentation)' 단계에서는 문법이나 표현, 어휘 등의 의미와 형태를 제시하고 교사의 설명을 통하여 학습자들을 이해할 수 있도록 한다. 그리고 '연습(Practice)' 단계에서는 제시 단계에서 설명한 내용을 학습자들이 내재화할 수 있도록 정확성(accuracy) 향상에 초점을 두고 수업을 구성한다. '활용(Use)' 단계에서는 학습자들이 담화 맥락에 맞게 목표 항목을 사용할 수 있도록 유창성(fluency) 향상에 도움을 줄 수 있는 과제(task)를 수행할 수 있도록 한다. 마지막으로 마무리(Follow up) 단계에서는 수업 목표를 다시 한번 상기시키고, 학습자들의 이해 정도를 점검하거나 다음 차시의 수업 계획과 함께 과제를 부여하는 것으로 수업을 구성하게 된다[8].

한국어 수업의 학습 단계의 구성과 내용을 살펴보면, 한국어 학습자들의 한국어 의사소통 능력 향상을 목적으로 한다는 점에서 의사소통 교수법(Communicative language learning)과 깊은 관련성을 가지고 있다[9]. 그리고 한국어 사용의 정확성을 높이기 위해 반복적이고 기계적인 학습을 진행한다는 측면에서 청각구두 교수법(Audio-lingual Method)과 관련성이 있다. 한국어 수업에서 해당 교수법이 구체적으로 드러나는 단계는 '연습' 단계와 '활용' 단계라고 할 수 있으며, '연습' 단계에서는 청각구두식 교수법의 특징이 나타나며, '활용' 단계에서는 의사소통 교수법의 특징이 강하게 드러난다[10].

연습 단계에서 적용하는 청각구두 교수법은 언어 사용에 대한 습관을 형성하기 위해 모방, 암기, 반복 등의 방법을 사용한다. 이를 위해 언어 사용의 정확성에 초점을 두고 교체, 대치, 변형, 완성 연습 등과 같은 '문형 연습(pattern practice)' 을 통하여 목표 항목을 익히도록 한다. 하지만 이러한 반복적인 학습과 기계적인 연습은 학습자의 흥미를 떨어뜨리고 목표 항목을 실제 언어 사용으로 전이시키는 데 한계를 가지고 있다. 이러한 문제점을 보완하기 위하여 활용 단계에서는 의사소통 교수법을 사용하여 실제적인 언어 사용에 중점을 두고 수업을 구성하여 진행하게 된다. 학습자가 실제 언어 상황에서 겪게 될 다양한 언어 상황을 고려하여 언어의 '형식' 보다는 '의미' 를 중심으로 학습자들이 언어 사용에 적극적으로 참여할 수 있도록 유도한다[11].

한편, 지금까지 이루어진 한국어 수업 관련 연구는 교사 교육과 교사 재교육 프로그램과 관련하여 강현화(2002), 최은규. 안경화(2003), 민현식(2005), 최주열(2008), 박지순 최진희(2010), 오경숙(2017), 방성원(2018) 등이 있으며 [8-14], 한국어 교육실습 교과목 운영과 관련하여 진정란(2014), 기준성(2015), 이윤진(2016), 이은경(2020) 등에서 연구가 이루어졌다[15-18]. 또한 한국어 수업 구성과 수업 계획안과 관련하여 진대연(2009), 장미라(2010), 민진영·최유하(2018), 김상수(2020), 구민지 · 박소연(2020) 등에서 논의가 이루어졌다[19-22]. 이상에서 살펴본 바와 같이 한국어 수업과 관련된 연구는 주로 한국어 교사 교육과 수업 운영 그리고 실습 교과목 운영에 필요한 내용과 방법을 주 대상으로 하였으며, 일부 연구에서 예비 교사의 수업 
구성과 내용에 대해 살피고 있다[23].

전문적이고 능숙한 한국어 교사를 배출하기 위해서는 예비 교사를 양성하는 단계에서부터 이들에게 수업 구성과 실행 능력을 갖출 수 있도록 지도해야 한다. 따라서 예비 교사가 한국어 수업을 구성하는 데 어떠한 어려움을 겪고 있으며, 그것을 해결하기 위해 이들에게 필요한 것이 무엇인지를 살피는 것은 반드시 필요한 과정이라고 할 수 있다.

\section{3. 연구 방법}

\section{1 자료 수집}

이 연구에 사용된 자료는 부산 소재 $A$ 대학의 평생교육원에서 운영하는 학점은행제 과정에서 '한국어교육실습' 교과목을 수강하는 수강생들이 작성한 한국어 수업지도안이다. '한국어교육실습' 교과목 이수 과정에서 수강생들은 수업지도안을 작성하게 되며 담당 교수로부터 이에 대한 피드백을 받게 된다. 수업지도안 작성은 개별적으로 한 학기에 3 회 실시하였으며, 작성한 수업지도안에 대한 피드백도 3 회 진행되었다. 수업지도안 작성 과정과 피드백 과정을 통하여 예비 교사들은 수업지도안 작성에 필요한 기본적인 사항부터 한국어 수업 구성과 내용에 대한 전반적인 사항에 대해 지도를 받을 수 있다.

따라서 예비 교사들을 지도하기 위해 작성한 수업지도안 피드백 보고서는 이들에게 나타난 수업지도안 작성의 문제점과 실태를 파악할 수 있는 자료가 될 수 있다. 자료는 2020년 3월 2020년 12월까지 수집되었으며, 5개 분반의 수강생 150명이 작성한 450편의 수업지도안에 대한 피드백 보고서이다. 전체 450편의 보고서 중에서 총 50 편의 보고서를 분석 대상으로 선정하였는데, 각 분반에서 최초 1 회차에 작성한 수업지도안에 대한 피드백 보고서 10편씩을 무작위로 선정하였다.

\section{2 연구 참여자}

이 연구에서 분석 대상으로 삼은 자료는 예비 한국어 교사 50 명이 작성한 한국어 수업지도안에 대한 피드백 보고서이다. 수업지도안 작성 지도는 5 명의 담당 교수에 의해 이루어졌으며, 담당 수강생들의 수업지도안에 대하여 피드백 보고서를 작성하였다. 수업지도안 지도에 대한 특별한 요구 사항은 없었으며, 수업지도안을 제출받은 후 3 일 이내에 피드백 내용을 작성하여 수강생과 연구자에게 이메일로 보내 줄 것을 요청하였다. 수업지도안 담당 교수자 5명은 모두 한국어교육학 박사과정을 수료하였으며, 한국어 교육 경력 10년 15년의 경력 교사이다. 이들은 한국어 교육 기관에서 교사들의 수업 관리와 교육과정 운영 등의 전반적인 교육 프로그램을 운영하고 있어서 수업지도안 작성 방법과 한국어 수업 구성, 내용 등에 대해서 충분한 사전 지식을 갖추고 있다.

\section{4. 연구 결과 및 논의}

수업지도안 피드백 보고서는 Nvivo12 프로그램을 사용하여 분석하였으며 분석결과, 전체 코딩 수는 228 개로 나타났다. 전체 코딩 항목은 수업 단계에 따라 재분류 
하였는데, 도입 단계 42개, 제시/설명 단계 86개, 연습 단계 48개, 활용 단계 46, 마무리 단계 6개' 로 나타났다. 제시/설명 단계에 대한 코딩 수가 86개로 가장 많이 나타났는데, 그 내용은 명시적인 문법 설명에 대한 요청과 문법 설명의 부족함에 대한 피드백, 문법 형태에 대한 설명 요청, 문법 설명 방법에 대한 문제 제기 등이다. 그리고 제시/설명 단계에서 어떤 내용을 판서나 PPT자료를 사용하여 학습자들에게 보여줄 것인지에 대한 문제점을 지적한 내용이 주를 이루었다. 수업 단계별 코딩 수와 내용을 정리하면 다음의 [표 1]과 같다.

[표 1] 수업 단계의 내용과 코딩 수

[Table 1] Class Construction Content and Number of Coding

\begin{tabular}{|c|c|c|c|}
\hline 수업단계 & 내용 & 코딩 수 & 비율 $(\%)$ \\
\hline 도입 단계 & $\begin{array}{c}\text { 명시적인 학습 목표 제시 } \\
\text { 동기유발 장치 마련 } \\
\text { 도입 단계 수업 구성의 부적절 } \\
\text { 도입의 수업 내용 부적절 } \\
\text { 학습자 수준에 맞지 않는 어휘나 문법 사용 } \\
\text { 목표 문법의 노출 방법 등 }\end{array}$ & 42 & 18.4 \\
\hline 제시/설명 단계 & $\begin{array}{c}\text { 명시적인 문법 설명 요청 } \\
\text { 문법 설명의 부족과 어려움 } \\
\text { 문법 형태에 대한 설명 요청 } \\
\text { 문법 설명 방법의 문제 } \\
\text { 판서나 PPT사용의 내용 등 } \\
\end{array}$ & 86 & 37.7 \\
\hline 연습 단계 & $\begin{array}{c}\text { 다양한 연습 활동 요청 } \\
\text { 연습 단계 전체에 대한 이해 부족 } \\
\text { 연습 단계에 맞지 않는 활동 } \\
\text { 연습 활동의 누락, 단계적인 연습 과제 제시 부족 등 }\end{array}$ & 48 & 21 \\
\hline 활용(사용) 단계 & $\begin{array}{c}\text { 사용단계 과제 활동의 다양성 부족, 사용단계 과제에 대한 이해 } \\
\text { 부족 } \\
\text { 사용단계 과제의 실제성 등 } \\
\end{array}$ & 46 & 20 \\
\hline 마무리 단계 & $\begin{array}{c}\text { 다음 차시 수업 예고 요청 } \\
\text { 정리 내용의 적절성 } \\
\text { 정리 단계 전체에 대한 이해 부족 등 }\end{array}$ & 6 & 2.6 \\
\hline & 합계 & 228 & 100 \\
\hline
\end{tabular}

\section{1 도입 단계}

도입 단계에서는 전 시간에 학습한 내용에 대해서 확인과 복습이 이루어지고 상황 맥락을 통하여 본 차시의 학습 목표와 내용을 자연스럽게 제시하고 학습자들의 동기를 유발하게 된다. 수업지도안의 도입 단계에 나타난 문제점을 살펴보면, 가장 많은 부분이 학습 목표를 명시적으로 제시하지 않는 경우가 많았다. 또한 도입 단계가 아니라 다음 수업 단계에서 학습자들에게 제시하고 설명해야 할 목표 문법을 도입 단계에서부터 설명하거나, 도입 단계에서 교사 발화에 목표 문법을 자연스럽게 노출시키지 못하는 문제점을 지적하는 보고서 내용을 확인할 수 있다. 이 외에도 도입 단계에서 학습자들의 학습 동기 유발을 위한 장치가 부족하거나 학습자 수준에 맞지 않는 어려운 표현을 사용하여 수업을 시작하고 진행하는 것이 보고되었다. 
도입에서는 목표 문법을 자연스럽게 노출하시고 이번 시간에 무엇을 배울지 학습 목표를 명시적으로 말씀해 주세요 (R15)

목표 문법의 의미 역시 교실에서 해도 되는 것과 안 되는 것을 몇 가지 예로 들어 말씀하시면 학생들이 쉽게 이해할 수 있습니다. 이렇게 자연스럽게 문형을 노출한 뒤에는 오늘 학습할 내용을 말씀해 주세요.(R23)

도입 단계에서는 학습자가 스스로 짐작하고 깨닫도록 유도하는 단계이므로 자세한 설명은 제시 설명 단계에서 사용하는 것이 좋습니다.(R38)

도입 단계에서 본시 학습 내용을 도입할 때는 학습자가 스스로 짐작할 수 있게 자료를 제시하거나 다양한 질문으로 학습자들의 동기 및 관심을 유발하도록 해야 합니다. 그런 의미에서 본 교안의 동기 부분이 조금 부족 합니다.(R42)

유물/유적은 중급 단계에서 배우는 단어입니다. 초급 수준에 맞게 단어를 사용해 주세요. 쉽게 교실이나 도서관 소재를 활용해서 문법을 노출시키기는 것이 좋습니다. (R46)

\section{2 제시/설명 단계}

제시/설명 단계에서는 목표 항목의 의미와 형태를 알려주고, 학습자들이 이해할 수 있도록 교사의 설명이 주를 이루게 된다. 전체 수업 구성에서 교사의 직접적인 역할이 가장 많이 나타나는 단계라고 할 수 있다. 수업지도안 피드백 보고서에 따르면 목표 문법에 대한 명시적인 설명과 형태 변화에 대한 설명이 이루어지지 않았음을 지적하거나, 목표 항목에 대한 제시와 설명이 다음 단계인 연습 단계에서 이루어지고 정작 이 단계에서는 제대로 이루어지지 않은 문제점을 지적하는 보고서 내용을 확인할 수 있다. 또한 목표 항목을 학습자들에게 쉽게 설명할 수 있는 방법이나 판서나 $\mathrm{PPT}$ 자료를 사용하여 목표 항목을 제시하고 설명하는 방법을 보고서에서 확인할 수 있었다.

제시/설명 단계에서 문법의 의미와 규칙을 설명해 주셔야 합니다. 연습 단계의 '규칙 설명지' 를 이 단계에서 보여 주세요.(R3)

제시 단계에서는 '았/었/였' 문법을 어떻게 사용하는지 설명하여야 합니다. 초급학생들이기 때문에 형태 변화와 불규칙 등도 자세히 설명해야 합니다. (R25)

선생님께서 제시하신 영상이 공공장소 예절에 맞기는 하지만 초급 학습자들이 듣고 이해하기에는 무리가 있습니다. 특히 초반 공공장소의 의미를 설명하는 부분은 영상이 아닌 교사의 설명으로 듣는 것이 더 좋을 것 같습니다. (R27)

이 단계에서는 학습 주제와 관련된 어휘와 목표 문법의 의미와 형태 규칙을 설명해 주세요. 이 단계에서 학습한 내용을 연습, 사용 단계에서 활용하는 것입니다. 공공장소의 예절과 관련된 어휘 '담배를 피우다, 쓰레기를 버리다, 물건을 만지다, 큰 소리로 떠들다, 음악을 크게 듣다' 등이 있습니다.(R30)

연습단계의 문법 형태 설명을 이 단계에서 해야 합니다. 이 단계에서 교사는 문법의 용법을 분명하게 보여 줄, 대표성이 있는 예문을 선별하여 판서하고 정확한 형태를 제시하는 것이 좋습니다. (R32)

'기숙사/요리하는 그림' , '도서관/이야기하는 그림' 등을 활용하여 목표 문법의 의미를 설명하면 학생들이 쉽게 이해할 것입니다.(R36) 
시각적인 자료를 제시하는 것이 학습자들이 내용을 이해하는 데 더 도움이 됩니다. 다음과 같이 목표 문법의 형태 규칙을 판서나 ppt 자료로 제시해 주세요.(R38)

\section{3 연습 단계}

연습 단계는 제시/설명 단계에서 교사의 설명을 통하여 학습한 내용을 학습자가 자신의 것으로 내재화하는 단계이다. 이 단계에서는 기계적이고 반복적인 연습과 유의적인 연습이 이루어진다. 따라서 학습자가 흥미를 가지고 다양한 연습을 할 수 있도록 내용을 구성하는 것이 필요하다. 피드백 보고서의 내용을 살펴보면, 기계적인 연습과 유의적인 연습이 제시될 수 있도록 요청하거나 연습 단계에 활용할 수 있는 활동을 구성하도록 요청하는 내용이 보고되었다. 특히, 예비 교사들이 연습 단계와 활용 단계를 잘 구분하지 못하여 학습 활동에 차이가 없거나 각 단계에 맞는 적절한 활동을 구성하지 못하는 문제점이 다수 보고되었다.

본 교안에서는 연습이 부족합니다. 연습 단계는 앞 단계에서 배운 내용에 대해 학습자가 내재화할 수 있도록 기계적인 연습과 유의미적인 연습을 단계적으로 제시하는 것이 좋습니다. (R2)

연습과 사용 부분에서 진행되는 활동이 거의 유사하여 학습자들이 지루함을 느낄 수 있습니다. 사용 부분에서 실제 의사소통 상황과 유사한 활동을 진행하는 것이 좋으며, 자유 회화, 상황극, 토론, 게임 등 다양한 활동이 가능합니다.(R7)

연습 단계의 활동과 활용 단계의 활동 유형이 거의 차이가 없습니다. 연습 단계는 교사가 주도하는 문법 설명을 들은 후 표현을 하기 위해 이해한 지식을 자기 것으로 내재화하고 자동화하는 훈련을 합니다. 연습 단계에서는 교체 연습, 응답 연습, 연결 연습, 문장 완성 연습 등을 할 수 있습니다. (R11)

다양한 공공장소의 예절을 말하는 것은 사용 단계에서 하는 건 어떨까요? 선생님께서 사용 단계에서 제시한 '교실 예절' 은 각 조에서 발표하기에는 중복되는 내용이 많을 것 같습니다. 그래서 교실 예절을 연습 단계에서 빈칸 채우기로 연습한 뒤, 다른 다양한 공공장소를 조별로 선택하여 예절을 말한다면 겹치는 내용이 조금은 줄 것 같습니다. (R15)

사진 자료를 활용하여 문장을 만들어 보는 연습 활동이 좋네요. 전 단계에서 노출되지 않은 다양한 어휘들을 활용할 수 있도록 자료를 제시해 주세요. 또한 '-아/어도 돼요' 를 활용하여 질문, 대답을 하는 연습 활동도 제시해 주시면 좋을 것 같습니다. (R25)

\section{4 활용(사용) 단계}

활용 단계에서는 연습 단계에서 정확성을 위해 연습한 내용을 바탕으로 유창성에 초점을 두고 과제를 수행하게 된다. 활용 단계에서는 과제 구성이 학습자의 의사소통 능력 향상에 적절한지를 살펴볼 필요가 있다. 또한 연습 단계의 과제와 활용 단계의 과제가 어떠한 차이가 있는지를 예비 교사들이 잘 인식하고 있는지 확인해볼 필요가 있다. 하지만 피드백 보고서에 따르면, 활용 단계에 대한 이해가 부족하여 이 단계에 적절한 활동을 구성하지 못하는 문제점이 가장 많이 나타났다. 
학습 목표는 공공장소의 예절입니다. 제시.설명 단계에서 학습한 내용과 연습 단계에서 연습한 것들을 모두 활용하여 사용 단계에서 실제와 가까운 언어 활동을 할 수 있도록 수업을 구성해 주세요. 공공장소를 하나씩 정해서 규칙/예절을 소개한다든지, 자신의 나라의 공공장소 예절을 소개한다든지, 예절을 지키지 않아 부끄러웠던, 불쾌했던 경험, 또는 짝과의 역할극 등의 활동을 활용할 수 있습니다. (R11)

제시·설명 단계나 연습 단계에서 언급되었던 내용이 반복적으로 사용되고 있어 학습자들의 흥미가 떨어질 것 같습니다. 그리고 제시하신 활동 역시 연습 단계와 크게 달라 보이지 않습니다. '사용' 단계는 실제 의사소통 상황과 최대한 가깝게 실제적인 활동이 이루어져야 합니다.(R17)

빈칸 채우기는 연습 단계에서 활용하기에 적당합니다. 사용 단계에서는 학습자들이 주도적으로 상황에 맞는 문장을 구성할 수 있도록 활동을 제시해 주세요. 각자 공공장소를 선택해서 규칙을 설명한다거나 규칙을 지키지 않아 미안하거나 불쾌했던 경험 등의 말하기가 좋습니다. (R19)

연습과 사용 활동이 교사 중심으로 이루어져 있습니다. 학습자가 주도적으로 활동에 참여할 수 있도록 수업을 구성해 주세요. 그리고 사용 단계에서는 단원의 주제 (공공장소)와 목표 문법(-아/어도 되다)을 실제 언어 상황에서 사용하는 단계입니다. 제시하신 사용 단계의 활동은 이에 맞지 않는 내용입니다.(R22)

사용 단계는 실제 생활에서 활용 가능한 유의미한 활동이 이루어져야 합니다. 제시된 활동은 그냥 질문에 대한 답일 뿐 학습자들이 목표 문법을 실제 생활에서 어떻게 사용할 수 있는지에 대한 연습이 되지 않습니다. (R36)

제시.설명 단계에서 학습한 내용과 연습 단계에서 연습한 것들을 모두 활용하여 사용 단계에서 실제와 가까운 언어 활동을 할 수 있도록 수업을 구성해 주세요. 경험, 또는 짝과의 역할극 등의 활동을 활용할 수 있습니다.(R39)

학습 목표는 공공장소의 예절입니다. 제시.설명 단계에서 학습한 내용과 연습 단계에서 연습한 것들을 모두 활용하여 사용 단계에서 실제와 가까운 언어 활동을 하 할 수 있도록 수업을 구성해 주세요. 공공장소를 하나씩 정해서 규칙/예절을 소개한다 든지, 자신의 나라의 공공장소 예절을 소개한다든지, 예절을 지키지 않아 부끄러웠던, 불쾌했던 경험, 또는 짝과의 역할극 등의 활동을 활용할 수 있습니다. (R50)

\section{5 마무리(정리) 단계}

마무리 단계에서는 수업의 전체적인 내용을 학습자가 어느 정도 이해했는지, 학업의 성취 정도를 확인하고 정리하게 된다. 마무리 단계와 관련된 보고서 내용은 다른 수업 단계와 비교했을 때 문제점이 가장 적게 나타났는데, 마무리 단계에서 교사들이 무엇을 해야 하는지에 대한 피드백 내용이 주를 이루었다.

수업 시간에 학습한 내용을 다시 한번 정리/확인해 주셔서 좋았습니다. 어휘뿐만 아니라 목표 문법도 함께 다루어 주시면 더욱 좋을 것 같습니다.(R6)

학습한 내용을 다시 한번 정리해 주셔서 좋습니다. 의미와 용법을 좀 더 구체적으로 말씀해 주셔도 좋을 것 같습니다.(R43)

새로 학습한 어휘와 문법 표현을 다시 한번 정리해 주세요. 학생들의 머릿속 에 더 오래 기억될 것입니다. 그리고 다음 시간에 학습할 내용도 예고해 주세요.(R48) 


\section{5. 결론}

이 연구에서는 예비 한국어 교사가 작성한 수업지도안에 대한 피드백 분석 보고서를 수집하여 예비 교사의 한국어 수업지도안 작성에 나타난 문제점과 실태를 파악하고자 하였다. 특히, 수업 구성과 내용을 탐색함으로써, 이들이 전공 교육과정을 통하여 학습한 전공 교과목에 대한 지식이 실제 현장에서 어떻게 실행될 수 있는지를 탐색하고, 그 과정에서 발생할 수 있는 문제점이 무엇인지 확인하고자 하였다.

수업지도안 피드백 보고서 50편에 나타난 내용을 수업 단계에 따라 분류하였으며, 그 결과 각 단계별 코딩 수는 '제시/설명-연습-활용-도입-마무리' 순으로 나타났다. 즉, 수업지도안을 작성하는 과정에서 예비 교사들은 제시/설명' 단계의 내용을 구성하는 데 가장 많은 어려움을 겪고 있었다. '제시/설명' 단계는 수업을 진행하는 과정에서 교사의 역할이 가장 크며, 교사의 목표 항목 제시와 설명에 따라 다음 단계의 수업 진행에 상당한 영향을 미칠 수 있다. 따라서 이 단계의 수업 구성에 어려움을 겪는 다는 것은 예비 교사들이 한국어 수업을 준비하는 과정에서 학습자의 수준이나 수업의 전체 목표에 따라 어떤 목표 항목을 제시하여야 하며, 설명을 어떻게 해야 하는지에 대한 이해가 부족하다는 것을 의미한다.

현장 경험이 없는 예비 교사가 이러한 어려움에 직면하는 것은 당연한 문제라고 생각 할 수 있으나, 전공과정을 이수하는 동안 한국어 교육 현장과 외국인 학습자를 이해할 수 있는 환경이 마련된다면 충분히 해결할 수 있는 문제점이라고 생각된다. 또한 예비 교사를 양성하는 교육기관과 교수자가 이러한 문제점을 인식하고 이를 전공 교과목 운영에 반영한다면 수업 설계와 계획 단계에서 직면하게 되는 예비 교사들의 고충과 어려움을 어느 정도 해결할 수 있는 있을 것이라 판단된다.

이 연구를 통하여 예비 한국어 교사가 수업지도안 작성 단계에서 겪는 어려움과 문제점을 확인하고 이들이 한국어 수업을 구성하고 실현하는 데 필요한 지식과 경험이 무엇인지에 대해서 논의하고자 하였다. 향후의 연구를 통하여 능숙하고 현장 적용 능력이 뛰어난 한국어 교사를 양성하기 위하여 한국어 수업 구성과 실현에 대한 구체적인 지도 방법을 제시한다면, 전문적이고 능숙한 한국어 교사 양성과 교육에 유용한 자료로 활용할 수 있을 것이다.

\section{References}

[1] M. J. Koo, S. Y. Park, Basic Research on Teaching of Lesson Planning in Korean Practicum Class, Journal of the International Network for Korean Language and Culture, (2020), Vol.17, No.2, pp.1-27.

[2] S. S. Kim, A Study on the Observation and Analysis of Korean Language Teachers' Classes, Urimal, (2020), No.63, pp.133-155, DOI: 10.35902/URM.2020.63.133

[3] J. C. Kang, A study on the Improvement Methods of Teaching Management Through the Systematic Classroom Activities Analysis, Journal of Educational Innovation Research, (2017), Vol.27, No.2, pp.221-250, DOI: 10.21024/pnuedi.27.2.201706.221

[4] H. J. Song, A Study on Korean Class Analysis For Instructional Consultation, Studies of Chinese \& Korean Humanities, (2015), No.46, pp.97-119, UCI: G704-000248.2015..46.006

[5] S. S. Kim, A Study on Class Observation for Class Diagnosis and Improvement, Asia-pacific Journal of Multimedia Services Convergent with Art, Humanities, and Sociology, (2018), Vol.8, No.1, pp.371-380, DOI: 
10.35873/ajmahs.2018.8.1.038

[6] S. M. Lee, A Research on class critique Through Korean Learner's class observation story, Korean Education, (2012), No.92, pp.447-470, DOI: 10.15734/koed..92.201209.447

[7] S. S. Kim, A Study on the Teachers' Perceptions of Error Correction, Asia-pacific Journal of Convergent Research Interchange, (2019), Vol.5, No.2, pp.1-9, DOI: 10.21742/apjcri.2019.06.01

[8] H. H. Kang, A Study of Needs Analysis in Program Development for Re-education of Korean Language Instructors Abroad, Journal of Korean Language Education, (2002), Vol.13, No.2, pp.21-38, UCI: G704-000597.2002.13.2.006

[9] E. G. Choi, G. H. An, A program development for Korean language teacher re-education: A case study for a Korean teacher re-education model for high school teachers in Japan, Journal of Korean Language Education, (2003), Vol.14, No.1, pp.323-341, UCI: G704-000597.2003.14.1.013

[10] H. S. Min, Korean Language Teacher Theory, The Role and Proper Characters of Korean Language Teachers in the 21st Century, Journal of Korean Language Education, (2005), Vol.16, No.1, pp.131-168, UCI: G704000597.2005.16.1.005

[11] J. Y. Choi, How to Nurture Korean Language Teachers and Elevate Their Status, Journal of Korean Language Education, (2008), Vol.19, No.1, pp.321-359, DOI: 10.18209/iakle.2008.19.1.321

[12] J. S. Park, J. H. Choi, A study on Korean teachers need of reeducation program for Korean teachers, Journal of Korean Language Education, (2010), Vol.21, No.3, pp.45-76, DOI: 10.18209/iakle.2010.21.3.45

[13] K. S. Oh, Case Studies of Developing Training Programs in Korea for Korean Language Instructors Overseas: With Focus on Training Programs for Korean Language Teachers at US K-12 Schools, Journal of Korean Language Education, (2017), Vol.28, No.2, pp.61-88, DOI: 10.18209/iakle.2017.28.2.61

[14] S. W. Bang, A Study on the Issues and Tasks of Korean Teachers' Reeducation, Journal of Korean Language Education, (2016), Vol.27, No.2, pp.79-103, DOI: 10.18209/iakle.2016.27.2.79

[15] J. R. Jin, A Case Study on the Practicum Class Management for Korean as a Foreign Language Education: Focus on the KFL Practicum class in Cyber University, Studies in foreign language education, (2014), Vol.28, No.2, pp.207-232, DOI: $10.16933 /$ sfle.2014.28.2.207

[16] J. S. Ki, A Study of Learners' Perception of Content and Methods of a Korean Practicum Class - Focusing on Class Observation, Journal of the International Network for Korean Language and Culture, (2015), Vol.13, No.2, pp.1-22, DOI: 10.15652/ink.2016.13.2.001

[17] Y. J. Lee, A study on the formulation and implementation of $<$ Model $1>$ in Practicum class for Korean as a foreign language: Based on the Korean education major courses in the graduate school of education, Bilingual Research, (2016), No.65, pp183-221, DOI: 10.17296/korbil.2016..65.183

[18] E. K. Lee, Study on the Practical Use of Peer Evaluation in The Mock-up Classes of Korean Education Practices, Teaching Korean as a Foreign Language, (2020) Vol.56, pp.353-374, DOI: 10.21716/TKFL.56.13

[19] D. Y. Jin, A Study on the Class Construction of KFL-Teachers, Korean Language Education Research, (2009), No.34, pp.429-466, DOI: 10.20880/kler.2009..34.429

[20] M. R. Jang, Research on Prospective Korean Teachers' Lesson Plan, The Korean Language and Literature, (2010), No.154, pp.57-91, DOI: 10.17291/kolali.2010..154.003

[21] J. Y. Min, Y. H. Choi, Analytical study of Korean Practicum for Students of Academic Credit Bank System Attendees -Focused on Mock Class-, Teaching Korean as a Foreign Language, (2018), Vol.49, pp.49-82, DOI: 10.21716/TKFL.49.49

[22] M. J. Koo, S. Y. Park, Basic Research on Teaching of Lesson Planning in Korean Practicum Class, Journal of the International Network for Korean Language and Culture, (2020), Vol.17, No.2, pp.1-27.

[23] S. S. Kim, M. K. Lee, A Study on the Instructional Design and Implementation of Korean Language Teachers, Asiapacific of Multimedia Services Convergent with art, Humanities, and Sociology, (2018), Vol.18, No.9, pp.583-591. 